\title{
ORIGINAL RESEARCH \\ Proton MR Spectroscopy Correlates of Frontal Lobe Function in Healthy Children
}

A. Ozturk

M. Degaonkar

M.A. Matson

C.T. Wells

E.M. Mahone

A. Horská
BACKGROUND AND PURPOSE: Neuroimaging methods have been used to improve our understanding of the topographic organization of the brain. In our study, proton ${ }^{1} \mathrm{H}-\mathrm{MR}$ spectroscopic imaging was used to evaluate frontal lobe function. The goal was to determine the relationship between neuropsychological measures of frontal lobe function and levels of a surrogate neuronal marker, $\mathrm{N}$-acetylaspartate (NAA), in typically developing healthy children and adolescents.

MATERIALS AND METHODS: Fifty-one healthy children (25 girls; 6.2-18.3 years of age; mean age, $12.3 \pm 3.6$ years) were examined. All children completed a neuropsychological assessment including measures of attention, executive function, memory, language, and visual and motor skills. ${ }^{1} \mathrm{H}-\mathrm{MR}$ spectroscopic imaging was performed by using a multisection spin-echo sequence at 1.5T. General linear model analysis of covariance was used to examine the relationship between the neuropsychological test scores and NAA/creatine $(\mathrm{Cr})$ ratios, controlling for age and sex.

RESULTS: A positive relationship between frontal lobe white matter NAA/Cr ratio and performance on 2 neuropsychological tests associated with frontal lobe function was detected. The Purdue Pegboard right-hand scores were higher with increasing NAA/Cr in the left frontal white matter $(P=.047)$, and Stanford-Binet-IV "Bead Memory" scores improved with increasing NAA/Cr ratio in the right frontal white matter $(P=.032)$.

CONCLUSIONS: An association between frontal white matter NAA/Cr ratios and 1) measures of manual speed and dexterity, and 2) visual working memory was detected. Our data may provide a quantitative basis for assessment of frontal lobe impairments in disease states.
$\mathbf{N}$ eurologic models of frontal lobe structure and function highlight 5 frontal-subcortical circuits, ${ }^{1,2}$ of which 2 are related to motor function and the other 3 are crucial in executive control. These circuits link specific regions of the frontal lobes to subcortical structures and supply technique-specific mechanisms for interaction with the environment. ${ }^{3}$ Motor and executive control systems develop in a parallel manner, such that both display a protracted developmental trajectory, with periods of rapid growth in elementary years and continued maturation into young adulthood. ${ }^{4}$ Working memory and response inhibition represent 2 core components of executive control that appear to be dissociable in children. ${ }^{5,6} \mathrm{Im}$ aging and lesion studies have linked working memory to the lateral prefrontal cortex. ${ }^{7,8}$ Frontal regions underlying response inhibition may differ, depending on the context in which the response needs to be inhibited. ${ }^{9}$

Compared with the adult brain, localization of function is less well-understood in children, due to continuing brain development. ${ }^{10-12}$ Understanding the relationship between neuropsychological function and frontal lobe neurochemistry in healthy children may help in the assessment of behavioral

Received November 26, 2008; accepted after revision February 3, 2009.

From the Russell H. Morgan Department of Radiology and Radiological Science (A.O., M.D., A.H.), Johns Hopkins University, Baltimore, Md; Children's Hospital Boston (M.A.M.), Boston, Mass; Pediatric Neuropsychology Division (C.T.W.), Children's National Medical Center, Washington, DC; and Department of Neuropsychology (E.M.M.), Kennedy Krieger Institute, Baltimore, Md.

This work was supported by National Institutes of Health Grants R01 NS042851, R 00052 , and P3OHD024061-16.

Please address correspondence to Alena Horská, PhD, Russell H. Morgan Department of Radiology and Radiological Science, Johns Hopkins University, 720 Rutland Ave, Traylor 217, Baltimore, MD 21205; e-mail: ahorska@jhmi.edu

Indicates open access to non-subscribers at www.ajnr.org

DOI 10.3174/ajnr.A1576 manifestations of neuronal dysfunction or impairment associated with pathologic levels of brain neurochemicals. Several neurochemicals related to brain tissue structure or metabolism can be detected by in vivo proton MR spectroscopy $\left({ }^{1} \mathrm{H}\right.$-MR spectroscopy). ${ }^{13}$ Application of ${ }^{1} \mathrm{H}$-MR spectroscopy to studies of the brain stimulated an interest in $\mathrm{N}$-acetylaspartate (NAA), a major chemical component of the central nervous system. NAA, which is found primarily in neuronal cells, can be considered a marker of neuronal function and integrity. $^{14}$

The purpose of this study was to determine the associations between the proposed neuropsychological measures of frontal lobe function and NAA levels in healthy children and adolescents by using high-resolution ${ }^{1} \mathrm{H}-\mathrm{MR}$ spectroscopic imaging. ${ }^{15}$ We hypothesized that performance-based measures of motor and executive control would be more highly correlated with frontal compared with nonfrontal NAA levels. We further hypothesized hemispheric specificity within these relationships, such that verbal working memory would be associated with left frontal NAA, whereas visuospatial working memory would correlate with right frontal NAA levels. Similarly, we hypothesized that right motor speed would correlate with left frontal NAA, whereas left motor speed would correlate with right frontal NAA levels.

\section{Materials and Methods}

The participants consisted of 51 healthy children and adolescents $(25$ female; 6.2-18.3 years of age; mean age, $12.3 \pm 3.6$ years; 40 righthanded). Participants were recruited by advertisement for a healthy typically developing control group for a research study. Children were initially screened via telephone interview with a parent and were excluded if there was a history of psychiatric or neurologic disorder, intellectual disability, language disorder, or learning disability. Additionally, participants were also excluded if they had an estimated in- 

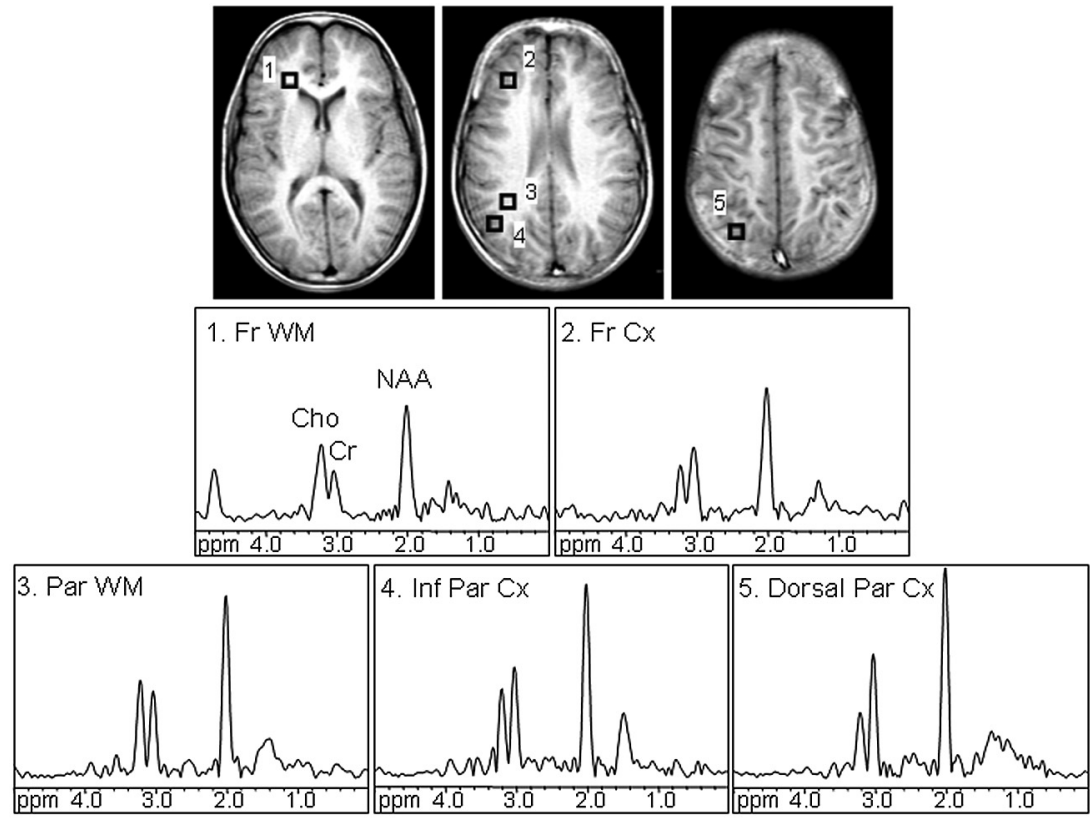

Fig 1. Axial T1-weighted spin-echo images display regions of interest in the frontal white matter (1, Fr WM), dorsolateral prefrontal cortex (2, Fr Cx), parietal white matter (3, Par WM), inferior parietal cortex (4, Inf Par Cx), and dorsal parietal cortex (5, Dorsal Par Cx). ${ }^{1} \mathrm{H}-\mathrm{MR}$ spectra corresponding to the evaluated regions of interest are shown. Signals of Cho, Cr, and NAA were detected. All spectra are equally scaled.

telligence quotient (IQ) of $<70$ based on administration of the Peabody Picture Vocabulary Test. ${ }^{16}$ Parents of those children meeting the eligibility criteria participated in a structured diagnostic interview using the Diagnostic Interview for Children and Adolescents-IV (DICA-IV), ${ }^{17}$ which is based on the Diagnostic and Statistical Manual of Mental Disorders, 4 th ed (DSM-IV). ${ }^{18}$ Children meeting DSM-IV criteria for a psychiatric disorder were subsequently excluded. Each family participating in the study signed a written informed consent form following the institutional review board standards of the Johns Hopkins Medical Institutions.

All participants completed a neuropsychological assessment, including measures of attention, executive function, memory, language, and visual and motor skills. Handedness was determined during the neuropsychological assessment by writing/drawing and by asking the child, during the Purdue Pegboard Test, ${ }^{19}$ which was his/ her preferred hand.

The tests were completed within 3 months of the ${ }^{1} \mathrm{H}-\mathrm{MR}$ spectroscopy examination. The following tests were used for the current analyses:

1. Purdue Pegboard (right and left hands), ${ }^{19}$ a measure of manual dexterity and fine-motor speed (corresponding functional domain: opposite motor cortex).

2. "Bead Memory" on the Stanford-Binet-IV (SB-IV), ${ }^{20}$ a visual working memory task (corresponding functional domain: right dorsolateral prefrontal cortex).

3. "Retrieval Fluency" (Woodcock Johnson Tests of Cognitive Abilities-III, WJ-III), ${ }^{21}$ a measure of rapid lexical retrieval, production, and efficient organization (corresponding functional domain: left supplementary motor cortex and dorsolateral prefrontal cortex).

4. "Auditory Working Memory" (WJ-III), a test of auditory (verbal) working memory (corresponding functional domain: left dorsolateral prefrontal cortex).

Routine brain MR imaging and ${ }^{1} \mathrm{H}-\mathrm{MR}$ spectroscopic imaging were performed at $1.5 \mathrm{~T}$. ${ }^{1} \mathrm{H}-\mathrm{MR}$ spectroscopic imaging was performed by using a multisection spin-echo sequence with outer-vol- ume suppression, ${ }^{15}$ with 4 supratentorial oblique axial 15 -mm-thick sections (2.5-mm gap) with TR/TE $=2000 / 140 \mathrm{~ms}$ and a nominal voxel size of $0.8 \mathrm{~mL}$. The full-echo signal was digitized with 256 data points, and a spectral width of $1000 \mathrm{~Hz}$ was used. Water suppression was accomplished with a single chemical shift selective pulse with a bandwidth of $110 \mathrm{~Hz}$. The ${ }^{1} \mathrm{H}-\mathrm{MR}$ spectroscopic datasets were processed by 3D Fourier transformation, with cosine filters in the spatial (phase-encoding) domains, after zero-filling to a $32 \times 32$ matrix size, and exponential line broadening of $3 \mathrm{~Hz}$, zero-filling to 8192 data points, and a high-pass convolution filter to remove the residual water signal intensity ( $50 \mathrm{~Hz}$ stop-band) in the time domain. No baseline correction was needed because the spectra obtained at the long TE had a flat baseline.

Signals of choline (Cho), creatine (Cr), and NAA were fitted to a gaussian line shape, applying a simplex routine, by using in-house software csx3 and imax3 (http://godzilla.kennedykrieger.org/csx/). Spectra were evaluated from the dorsolateral prefrontal cortex, frontal white matter (forceps minor), dorsal and inferior parietal cortex, and deep parietal white matter in both hemispheres (Fig 1). Only voxels that appeared to be completely encompassed in the desired region of interest on the localizer images were included for the analyses. The NAA/Cr ratio was calculated from areas under the respective signals. Data from 2-4 voxels for each region were averaged. Before ${ }^{1} \mathrm{H}-\mathrm{MR}$ spectroscopic imaging, T1-weighted localizer images were recorded at the same section location and thickness as ${ }^{1} \mathrm{H}-\mathrm{MR}$ spectroscopic imaging for selection of the regions of interest.

A paired $t$ test was used to assess differences in NAA/Cr between the hemispheres. Linear regression analysis was used to test the effect of age on NAA/Cr and on neuropsychological tests scores. Raw scores of neuropsychological tests were used in all analyses. General linear model analysis of covariance (ANCOVA) was applied to evaluate the relationship between the neuropsychological test scores and the NAA/Cr ratio, controlling for sex. Additional ANCOVA analyses were performed to include race, handedness, and socioeconomic status. Neuropsychological test raw scores and NAA/Cr ratios were log- 


\begin{tabular}{|c|c|c|c|c|c|c|}
\hline & \multicolumn{2}{|c|}{$\begin{array}{c}\text { Boys } \\
(n=26) \\
\end{array}$} & \multicolumn{2}{|c|}{$\begin{array}{c}\text { Girls } \\
(n=25)\end{array}$} & \multicolumn{2}{|c|}{$\begin{array}{c}\text { Total } \\
(n=51)\end{array}$} \\
\hline & Mean & SD & Mean & SD & Mean & SD \\
\hline$\overline{\text { Age }(y r)}$ & 12.0 & 3.9 & 12.6 & 3.3 & 12.3 & $\overline{3.6}$ \\
\hline SES & 45.9 & 11.3 & 42.9 & 13.3 & 44.5 & 12.2 \\
\hline Estimated IO & 139 & 31 & 142 & 27 & 141 & 29 \\
\hline \multicolumn{7}{|c|}{ Neuropsychological test scores } \\
\hline Purdue Pegboard RH & 12.7 & 2.1 & 13.3 & 2.3 & 13.0 & 2.2 \\
\hline Purdue Pegboard LH & 11.9 & 2.5 & 13.3 & 2.6 & 12.6 & 2.6 \\
\hline SB-IV "Bead Memory" & 22.8 & 4.9 & 24.4 & 5.4 & 23.6 & 5.2 \\
\hline $\begin{array}{l}\text { WJ-III "Auditory Working } \\
\text { Memory" }\end{array}$ & 21.1 & 9.1 & 22.5 & 6.1 & 21.8 & 7.8 \\
\hline WJ-III "Retrieval Fluency" & 56.1 & 15.1 & $67.4^{*}$ & 17.1 & 61.9 & 16.9 \\
\hline \multicolumn{7}{|l|}{$\mathrm{NAA} / \mathrm{Cr}$} \\
\hline \multirow[t]{2}{*}{ Frontal GM } & R 2.1 & 0.5 & 2.1 & 0.4 & 2.1 & 0.5 \\
\hline & L 2.2 & 0.4 & 2.0 & 0.3 & 2.1 & 0.3 \\
\hline \multirow[t]{2}{*}{ Frontal WM } & R 2.5 & 0.3 & 2.5 & 0.3 & 2.5 & 0.3 \\
\hline & L 2.4 & 0.3 & 2.4 & 0.3 & 2.4 & 0.3 \\
\hline \multirow[t]{2}{*}{ Parietal GM } & R 2.1 & 0.2 & 2.1 & 0.2 & 2.1 & 0.2 \\
\hline & L 2.1 & 0.2 & 2.1 & 0.2 & 2.1 & 0.2 \\
\hline \multirow{2}{*}{ Parietal WM } & R 2.5 & 0.4 & 2.6 & 0.3 & 2.5 & 0.4 \\
\hline & L 2.3 & 0.3 & 2.5 & 0.2 & 2.4 & 0.3 \\
\hline
\end{tabular}

Note:- $R$ indicates right; $L$, left; $L H$, left hand; $R H$, right hand; WM, white matter; GM, gray matter; SES, socioeconomic status; NAA/Cr, $\mathrm{N}$-acetylaspartate/creatine ratio. *Girls had higher scores on the WJ-III "Retrieval Fluency" test $(P<.05)$.

\begin{tabular}{|c|c|c|c|c|c|c|}
\hline & \multicolumn{2}{|c|}{ Boys $(n=26)$} & \multicolumn{2}{|c|}{ Girls $(n=25)$} & \multicolumn{2}{|c|}{ Total $(n=51$} \\
\hline & No. & $\%$ & No. & $\%$ & No. & $\%$ \\
\hline \multicolumn{7}{|l|}{ Race } \\
\hline Caucasian & 13 & 25 & 8 & 16 & 21 & 41 \\
\hline African American & 10 & 20 & 15 & 29 & 25 & 49 \\
\hline Other & 3 & 6 & 2 & 4 & 5 & 10 \\
\hline \multicolumn{7}{|l|}{ Handedness } \\
\hline Right & 19 & 37.3 & 21 & 41.2 & 40 & 78 \\
\hline Left & 7 & 13.7 & 4 & 7.8 & 11 & 22 \\
\hline Mixed & 0 & 0 & 0 & 0 & 0 & 0 \\
\hline
\end{tabular}

transformed, and the residuals, after regressing on age, were calculated to account for heteroscedasticity ${ }^{22}$ and for the effect of the age on both dependent and independent variables. Control analyses between neuropsychological test scores and NAA/Cr ratios in a region where no relationship was expected (parietal lobe gray and white matter) were also performed. In all statistical analyses, the significance level was set to $P<.05$.

\section{Results}

Figure 1 shows T1-weighted spin-echo localizer images depicting the regions of interest and corresponding spectra. High-resolution good-quality spectra were obtained in most subjects; a small number of spectra could not be processed due to susceptibility or motion artifacts ( $n \leq 3$ in each region). Tables 1 and 2 list demographic information, raw scores of the neuropsychological tests, and mean lobar gray and white matter NAA/Cr ratios.

Raw scores of all tests improved with age (all, $P<.0002$; $\left.0.2 \leq r^{2} \leq 0.54\right)$. Girls had $20 \%$ higher scores than boys on the WJ-III "Retrieval Fluency" test $(P=.008)$; no other sex differences in neuropsychological test scores were detected.

NAA/Cr tended to increase with age in the right parietal white matter (slope $=0.039$ year $^{-1}, P=.018, r^{2}=0.12$ ) and the right parietal gray matter (slope $=0.011$ year $^{-1}, P=.059$, $\left.r^{2}=0.08\right)$. No differences in the NAA/Cr ratio between the hemispheres were detected in any of the examined regions. The only detected sex difference was a $0.2 \%$ higher NAA/Cr ratio in the right frontal white matter in girls than in boys $(P=$ $.011)$.

Of the tests hypothesized to be associated with the left frontal lobe, the Purdue Pegboard right-hand raw scores improved with increasing left frontal white matter NAA/Cr ratios, controlling for age and sex $(P=.047)$ (Fig 2A). No significant relationship between the Purdue Pegboard right-hand raw scores and left frontal gray matter NAA/Cr ratios was detected. ANCOVA did not reveal any significant relationship between the raw scores of the WJ-III "Auditory Working Memory" or WJ-III "Retrieval Fluency" and left frontal gray or white matter NAA/Cr ratios. As expected, there were no significant relationships between the tests hypothesized to be associated with right frontal lobe function (Purdue Pegboard left hand, "Bead Memory") and left frontal gray or white matter NAA/Cr ratios (all, $P>.2$ ).

Of the tests hypothesized to be associated with the right frontal lobe, the SB-IV "Bead Memory" raw scores improved with increasing right frontal white matter NAA/Cr ratios $(P=$ .032) (Fig 2B). No significant relationship between the SB-IV "Bead Memory" raw scores and right frontal gray matter NAA/Cr ratios was detected. ANCOVA did not detect any significant relationship between the Purdue Pegboard lefthand raw scores or tests of left frontal function (Purdue Pegboard right hand, WJ-III "Auditory Working Memory," WJ-III "Retrieval Fluency") and right frontal gray or white matter NAA/Cr ratios (all, $P>.2$ ).

No significant effect on race, handedness, or socioeconomic status was detected in the analyses showing a significant relationship between the neuropsychological variables and NAA/Cr. The control analyses did not reveal any significant relationship between raw scores of any of the tests hypothesized as associated with frontal lobe function and parietal gray or white matter NAA/Cr ratios.

\section{Discussion}

To appreciate the extent of cognitive damage caused by brain pathology or injury, one must evaluate localization of brain function in typically developing brains. ${ }^{1} \mathrm{H}-\mathrm{MR}$ spectroscopy is a sensitive method to detect normal variation in brain functioning. ${ }^{23}$ Our study, performed in a cohort of carefully screened healthy children and adolescents, demonstrated a positive relationship between frontal lobe white matter NAA/Cr ratio and scores of 2 neuropsychological tests associated with frontal lobe function - the Purdue Pegboard right hand (a test assessing manual dexterity and speed) and SB-IV "Bead Memory" test (a test of visuospatial working memory). The scores on the Purdue Pegboard right-hand test improved with increasing left frontal white matter NAA/Cr ratios, and the scores of the SB-IV "Bead Memory" test improved with increasing right frontal white matter NAA/Cr ratios. Our results suggest that ${ }^{1} \mathrm{H}-\mathrm{MR}$ spectroscopy can provide a means for testing specific hypotheses on the relationship between brain function and neurochemistry.

During the past 2 decades, ${ }^{1} \mathrm{H}$-MR spectroscopy has been used in patients with a wide range of neurologic and psychiat- 

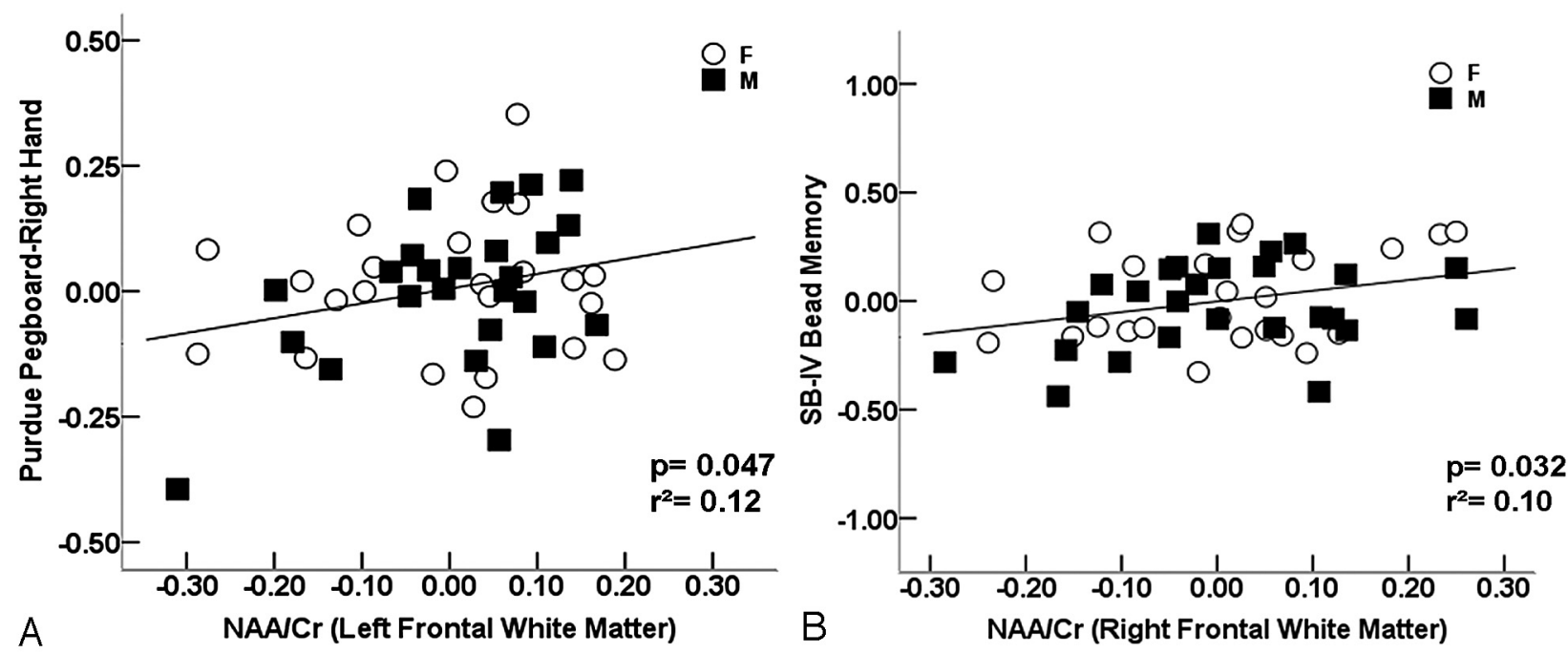

Fig 2. Scatterplots showing the relationship between the NAA/Cr ratio in the left frontal white matter and scores of Purdue Pegboard right hand $\left(\right.$ slope $=0.611$ year $\left.{ }^{-1}, P=.047\right)(A)$ and between the NAA/Cr ratio in the right frontal white matter and SB-IV "Bead Memory" (slope $=0.494$ year $\left.^{-1}, P=.032\right)(B)$. Residuals of the test scores and NAA/Cr after regressing on age are plotted.

ric disorders to understand the effects of brain pathologies, identify differences between diagnostic groups, and monitor long-term changes with or without drug therapy. ${ }^{23}$ Abnormal frontal lobe NAA levels have been reported in children with traumatic brain injury, ${ }^{24,25}$ lead exposure, ${ }^{26}$ epilepsy, ${ }^{27}$ developmental delay, ${ }^{28}$ bipolar disorder, ${ }^{29}$ psychosis, ${ }^{30}$ autism, ${ }^{31}$ and childhood obstructive sleep apnea. ${ }^{32}$ Frontal NAA levels were directly related to performance on language and visuomotor tests, ${ }^{24}$ mathematic skills, ${ }^{25}$ and reaction times ${ }^{33}$ in children with traumatic brain injury. In a group of healthy 20to 75-year-old women, NAA in the dorsolateral prefrontal cortex positively correlated with vocabulary scores. ${ }^{34}$ A significant correlation between frontal white matter NAA levels and a composite measure of speed of information processing, attention, and visual memory was observed in healthy elderly subjects. ${ }^{35}$ In a recent review, previous findings on the application of MR spectroscopy in cognitive research were summarized. ${ }^{23}$ It was noted that data in healthy controls were limited. Only a few studies have evaluated the relationship between NAA levels and neuropsychological performance in healthy adolescents $^{36}$ or children. ${ }^{10}$

NAA, believed to be a marker of neuronal health and function, has been a compound of major interest in neuropsychological studies. In several of the studies including patients with brain pathologies, levels of other neurochemicals, such as $\mathrm{Cho}^{24,25,33,37,38}$ or myo-inositol (mIns) ${ }^{39,40}$ were reported to be associated with the degree of cognitive impairment. However, NAA levels may have the strongest and most consistent correlations with cognitive outcome measures, ${ }^{41}$ particularly in the healthy brain. ${ }^{23}$ Jung et $\mathrm{al}^{42}$ reported a positive correlation between occipitoparietal white matter NAA and full-scale IQ (FSIQ) and a negative correlation between Cho and FSIQ in healthy young adults. The results on NAA but not on Cho were confirmed in a later study. ${ }^{43}$ No specific hypotheses on the relationship between Cho levels and neuropsychological performance were considered in our study. We note, however, that the exploratory analyses we performed did not show any evidence of a significant relationship between regional gray and white matter Cho/Cr ratios and neuropsychological test scores (data are not reported).

The frontal lobe is associated with sustained attention, executive function, and memory retrieval. ${ }^{44}$ Frontal lobes have the highest degree of connectivity and are involved in integration of mental operations. ${ }^{44}$ Most previous research studies on higher cognitive abilities examined the cerebral cortex. However, more recent studies demonstrated that maturation of white matter is associated with development of specific cognitive abilities in children and adolescents and that white matter abnormalities are associated with a wide spectrum of cognitive and emotional problems. ${ }^{44,45}$ Advances in research on white matter were facilitated by introduction of novel techniques of physiologic neuroimaging. These techniques, including ${ }^{1} \mathrm{H}-$ MR spectroscopy, were instrumental in improving our understanding of the relationship between white matter structure and brain function across the lifespan in healthy and diseased states. $^{12}$ In healthy participants, age-related differences in frontal white matter metabolism were related to age-related differences in cognition. ${ }^{35,46}$ A positive correlation between NAA levels in the left frontal subcortical white matter and performance on executive and attentional tasks ${ }^{46}$ and a composite measure of speed of information processing was reported in healthy middle-aged and elderly adults. ${ }^{35}$ In a larger group of middle-aged and elderly adults, a positive correlation between NAA levels in the centrum semiovale and executive functioning (but not working memory, information-processing speed, long-term memory, or fluid intelligence) was detected. ${ }^{47}$ In addition, measures of water diffusion in the tissue (fractional anisotropy and mean diffusivity) obtained with diffusion tensor imaging (DTI) correlated with working memory (but not executive function and information processing speed). ${ }^{48}$ Our results in children, showing higher white matter NAA/Cr ratios with higher scores on both the Purdue Pegboard and SB-IV "Bead Memory" tests, are in accord with a previously demonstrated association between white matter maturity and cognitive abilities, particularly those involving motor speed and executive functions. 
The Purdue Pegboard Test is a relatively simple task involving motor speed. Functional MR imaging (fMRI) showed that in healthy subjects (including children), finger movements with either hand primarily activate the sensorimotor and premotor cortices in both hemispheres, with a predominant activation of the contralateral hemisphere. ${ }^{49-51}$ The range of raw scores for the Purdue Pegboard Test was in general agreement with those reported in the National Institutes of Health MR imaging study on normal brain development, ${ }^{52}$ though in our sample, girls did not have higher scores than boys. As expected for the age of our participants, the overall disparity between hands was small. For the Purdue Pegboard Test, the only significant correlation was detected for the right hand, which was dominant for most of our subjects (40 of 51). Although both Purdue Pegboard left-hand and right-hand test scores and left and right frontal white matter NAA/Cr ratios were highly correlated (both $P<.0001$ ), we did not find a significant correlation between the Purdue Pegboard left hand and right frontal white matter NAA/Cr ratios. This finding may be due to a larger scatter of scores for the left-hand test, observed mainly in boys.

Several other ${ }^{1} \mathrm{H}-\mathrm{MR}$ spectroscopy studies compared scores on the Purdue Pegboard or Grooved Pegboard tests and NAA, mIns, or Cho levels in diseased states and healthy controls. However, these studies did not state specific hypotheses regarding expected correlations in specific brain regions and involved a relatively small number of subjects. ${ }^{37,39}$ A strong positive correlation $(P=.006)$ between the Purdue Pegboard dominant test scores and right frontal white matter mIns/Cr ratios and a correlation between the Purdue Pegboard nondominant test scores and mIns concentration in the right hippocampal region $(P=.03)$ were detected in 13 healthy control subjects 6-16 years of age. ${ }^{37}$ If most of the children were righthanded (handedness was not reported), the significant correlation with the ipsilateral hemisphere appears unexpected. No significant relationship between the Purdue Pegboard right and left hands and left medial temporal lobe or left basal ganglia was detected in 20 healthy young adults. ${ }^{53}$

SB-IV "Bead Memory" test is a task of visual working memory, a short-term maintenance and manipulation of visual information. In our sample of healthy controls, the range of raw scores for the "Bead Memory" test was consistently within the average range for age, based on age-based norms from the SB-IV standardization manual. ${ }^{20}$ In humans, areas specialized for object and spatial working memory are located in the prefrontal cortex. ${ }^{54}$ Previous studies demonstrated hemispheric laterality, with a left midfrontal region specialized for object working memory and right superior and inferior frontal regions specialized for spatial working memory. ${ }^{55}$ The importance of frontal white matter maturation for the development of visuospatial working memory networks, including frontoparietal cortical regions and connecting white matter tracts, was also demonstrated in DTI studies of white matter in children and adolescents. ${ }^{56}$ In agreement with these findings, we detected a significant relationship between the SB-IV "Bead Memory" test scores and NAA/Cr ratios in the right frontal white matter and not in the left frontal white matter. Similar to those in our study, NAA levels in the right frontal white matter were associated with visual working memory (Visual 2-Back Test) in 7- to 12-year-old children. ${ }^{10}$

A relationship between NAA/Cr and verbal working memory performance was also tested in our study. A recent study in patients with focal lesions in the inferior frontal or inferior parietal lobe examined specific roles of these 2 brain regions in verbal working memory. ${ }^{57}$ In a group of healthy adults and patients with schizophrenia, verbal learning task performance was positively correlated with the NAA/Cr ratio in the left inferior frontal cortex. ${ }^{58}$ However, the correlation was not significant in either group alone. A lower NAA/Cho ratio in the right frontal cortex (and left hippocampal region) has been reported in children with obstructive sleep apnea (compared with healthy children), who also demonstrated associated deficits in verbal working memory and verbal fluency ${ }^{32}$; however, no correlations were reported.

In our study, no significant relationship between the scores on the verbal working memory tests and frontal or parietal $\mathrm{NAA} / \mathrm{Cr}$ ratios was detected. A possible explanation may be that the WJ-III "Auditory Working Memory" is not as direct a measure of frontal lobe function as the Purdue Pegboard or the "Bead Memory." It has been reported that WJ-III "Auditory Working Memory" was moderately correlated with frontal lobe gray matter volumes but also moderately correlated with temporal and parietal white matter volumes, suggesting that it may be functionally less specific than other tests because of broader representation of language in children. ${ }^{59}$ Similarly, the WJ-III "Retrieval Fluency" test may be less specific to frontal lobe function, with a more broad representation in the brain (frontal and temporal regions).

${ }^{1} \mathrm{H}-\mathrm{MR}$ spectroscopy has been an important tool in the diagnosis and treatment of many disorders affecting the pediatric brain, such as epilepsy, metabolic diseases, neoplasms, hypoxic-ischemic insults, and childhood neurodegenerative and neuropsychiatric disorders. ${ }^{60,61}$ Clinical applications of ${ }^{1} \mathrm{H}$-MR spectroscopy are being rapidly developed in the field of pediatric neuropsychiatric disorders because of the lack of robust findings on conventional imaging in general. However, the underlying pathologies may be revealed by methods of physiologic imaging, including ${ }^{1} \mathrm{H}-\mathrm{MR}$ spectroscopy, fMRI, and DTI. ${ }^{62}$ Although our analyses were focused on the neuronal marker, NAA, it has to be noted that NAA may not be affected in all pathologies or patients (for example, in bipolar affective disorder ${ }^{63}$ or depression ${ }^{64}$ ).

There are several limitations to our study. First, the subject group consisted of 51 participants. Therefore, with larger samples, future studies may show statistically significant correlations in additional comparisons. Significant results were not detected in all hypothesized relationships, and only frontal brain regions were examined. There was also a relatively wide age range for the number of subjects included. Thus, it may not be possible to evaluate how consistent our results are across the entire age range of $6-18$ years. ${ }^{1} \mathrm{H}-\mathrm{MR}$ spectroscopy data were collected with a high-resolution spectroscopic imaging technique, minimizing partial volume effects. However, metabolite concentrations were not measured and NAA levels were expressed as NAA/Cr ratios. Variability in NAA/Cr ratios may, therefore, be explained not only by differences in NAA concentrations but also by differences in $\mathrm{Cr}$ concentrations. 


\section{Conclusions}

Our data contribute to a growing body of literature on the association between neuropsychological test scores and neurometabolites. ${ }^{23}$ Most of the previous studies evaluating correlations between MR spectroscopy-detected metabolites and cognitive measures were focused on specific diseases; as stated above, there have been few reports in healthy children or adults. ${ }^{23,35,36}$ Our population included thoroughly screened subjects, by using a detailed psychiatric interview, parents' ratings, and a detailed neuropsychological test battery. Individual test scores, rather than composite scores (including similar tests used in our study), ${ }^{24,41,65,66}$ were used. Since frontal lobe white matter is affected by a number of brain pathologies, our data may provide a quantitative basis for assessment of frontal lobe impairments in disease states. Future research will be important in determining the relationship between NAA/Cr ratios and neuropsychological test performance in clinical populations (including those children with congenital and acquired conditions), and if there are pathologies associated with abnormal NAA levels but normal neuropsychological test scores (or vice versa).

\section{Acknowledgments}

We thank Amanda Barnes, Rena Geckle, and Cynthia Schultz for their help with recruiting the subjects and performing the MR imaging examinations.

\section{References}

1. Burruss JW, Hurley RA, Taber KH, et al. Functional neuroanatomy of the frontal lobe circuits. Radiology 2000;214:227-30

2. Lichter JB, Cummings JL. Introduction and overview. In: Lichter DG, Cummings JL, eds. Frontal-Subcortical Circuits in Psychiatric and Neurological Disorders. New York: Guilford Press; 2001:1-43

3. Castellanos FX, Sonuga-Barke EJ, Milham MP, et al. Characterizing cognition in ADHD: beyond executive dysfunction. Trends Cogn Sci 2006;10:117-23

4. Diamond A. Close interrelation of motor development and cognitive development and of the cerebellum and prefrontal cortex. Child Dev 2000;71:44-56

5. Goldberg MC, Mostofsky SH, Cutting LE, et al. Subtle executive impairment in children with autism and children with ADHD. J Autism Dev Disord 2005;35:279-93

6. Mahone EM, Pillion JP, Hoffman J, et al. Construct validity of the auditory continuous performance test for preschoolers. Dev Neuropsychol 2005;27:11-33

7. D'Esposito M, Postle BR, Rypma B. Prefrontal cortical contributions to working memory: evidence from event-related fMRI studies. Exp Brain Res 2000;133:3-11

8. Mull BR, Seyal M. Transcranial magnetic stimulation of left prefrontal cortex impairs working memory. Clin Neurophysiol 2001;112:1672-75

9. Mostofsky SH, Schafer JG, Abrams MT, et al. fMRI evidence that the neural basis of response inhibition is task-dependent. Brain Res Cogn Brain Res 2003;17:419-30

10. Yeo RA, Hill D, Campbell R, et al. Developmental instability and working memory ability in children: a magnetic resonance spectroscopy investigation. Dev Neuropsychol 2000;17:143-59

11. Davidson MC, Thomas KM, Casey BJ. Imaging the developing brain with fMRI. Ment Retard Dev Disabil Res Rev 2003;9:161-67

12. Paus T, Collins DL, Evans AC, et al. Maturation of white matter in the human brain: a review of magnetic resonance studies. Brain Res Bull 2001;54:255-66

13. Barker PB, Lin DDM. In vivo proton MR spectroscopy of the human brain. Prog Nucl Magn Reson Spectrosc 2006;49:99-128

14. Barker PB. N-acetyl aspartate: a neuronal marker? Ann Neurol 2001;49:423-24

15. Duyn JH, Gillen J, Sobering G, et al. Multisection proton MR spectroscopic imaging of the brain. Radiology 1993;188:277-82

16. Dunn LM. Peabody Picture Vocabulary Test. 3rd ed. Bloomington, Minn: Pearson Assessments; 1997

17. Reich W, Welner Z, Hernajic B. The Diagnostic Interview for Children and Adolescents-IV. North Tonawanda, NY: Multi-Health Systems; 1997

18. Diagnostic and Statistical Manual of Mental Disorders, 4th ed. Washington, DC: American Psychiatric Association; 1994
19. Tiffin J. Purdue Pegboard: Examiner's Manual. Chicago, Ill: Science Research Associates; 1968

20. Thorndike RM, Hagen EP, Sattler JM. Stanford-Binet Intelligence Scales. Itasca, Ill: Riverside Publishing; 1986

21. Woodcock RW, McGrew KS, Mather N. Woodcock-Johnson III. Itasca, Ill: Riverside Publishing; 2001

22. Zar J. Biostatistical Analysis. 4th ed. Upper Saddle River, NJ: Prentice Hall; 1999:356

23. Ross AJ, Sachdev PS. Magnetic resonance spectroscopy in cognitive research. Brain Res Brain Res Rev 2004;44:83-102

24. Yeo RA, Phillips JP, Jung RE, et al. Magnetic resonance spectroscopy detects brain injury and predicts cognitive functioning in children with brain injuries. J Neurotrauma 2006;23:1427-35

25. Hunter JV, Thornton RJ, Wang ZJ, et al. Late proton MR spectroscopy in children after traumatic brain injury: correlation with cognitive outcomes. $A J N R$ Am J Neuroradiol 2005;26:482-88

26. Trope I, Lopez-Villegas D, Cecil KM, et al. Exposure to lead appears to selectively alter metabolism of cortical gray matter. Pediatrics 2001;107:1437-42

27. Simister RJ, McLean MA, Barker GJ, et al. Proton MRS reveals frontal lobe metabolite abnormalities in idiopathic generalized epilepsy. Neurology 2003;61:897-902

28. Filippi CG, Ulug AM, Deck MD, et al. Developmental delay in children: assessment with proton MR spectroscopy. AJNR Am J Neuroradiol 2002;23:882-88

29. Olvera RL, Caetano SC, Fonseca M, et al. Low levels of $\mathrm{N}$-acetyl aspartate in the left dorsolateral prefrontal cortex of pediatric bipolar patients. JChild Adolesc Psychopharmacol 2007;17:461-73

30. Zabala A, Sanchez-Gonzalez J, Parellada M, et al. Findings of proton magnetic resonance spectrometry in the dorsolateral prefrontal cortex in adolescents with first episodes of psychosis. Psychiatry Res 2007;156:33-42

31. Kleinhans NM, Schweinsburg BC, Cohen DN, et al. N-acetyl aspartate in autism spectrum disorders: regional effects and relationship to fMRI activation. Brain Res 2007;1162:85-97

32. Halbower AC, Degaonkar M, Barker PB, et al. Childhood obstructive sleep apnea associates with neuropsychological deficits and neuronal brain injury. PLoS Med 2006;3:e301

33. Parry L, Shores A, Rae C, et al. An investigation of neuronal integrity in severe paediatric traumatic brain injury. Child Neuropsychol 2004;10:248-61

34. Pfleiderer B, Ohrmann P, Suslow T, et al. N-acetylaspartate levels of left frontal cortex are associated with verbal intelligence in women but not in men: a proton magnetic resonance spectroscopy study. Neuroscience 2004;123:1053-58

35. Ross AJ, Sachdev PS, Wen W, et al. Cognitive correlates of 1H MRS measures in the healthy elderly brain. Brain Res Bull 2005;66:9-16

36. Gimenez M, Junque C, Narberhaus A, et al. Medial temporal MR spectroscopy is related to memory performance in normal adolescent subjects. Neuroreport 2004;15:703-07

37. Keller MA, Venkatraman TN, Thomas A, et al. Altered neurometabolite development in HIV-infected children: correlation with neuropsychological tests. Neurology 2004;62:1810-17

38. Colla M, Ende G, Bohrer M, et al. MR spectroscopy in Alzheimer's disease: gender differences in probabilistic learning capacity. Neurobiol Aging 2003;24:545

39. Chang L, Ernst T, Witt MD, et al. Relationships among brain metabolites, cognitive function, and viral loads in antiretroviral-naive HIV patients. $\mathrm{Neu}$ roimage 2002;17:1638

40. Olson BL, Holshouser BA, Britt W 3rd, et al. Longitudinal metabolic and cognitive changes in mild cognitive impairment patients. Alzheimer Dis Assoc Disord 2008;22:269-77

41. Babikian T, Freier MC, Ashwal S, et al. MR spectroscopy: predicting long-term neuropsychological outcome following pediatric TBI. J Magn Reson Imaging 2006;24:801-11

42. Jung RE, Brooks WM, Yeo RA, et al. Biochemical markers of intelligence: a proton MR spectroscopy study of normal human brain. Proc Biol Sci 1999;266:1375-79

43. Jung RE, Haier RJ, Yeo RA, et al. Sex differences in $\mathrm{N}$-acetylaspartate correlates of general intelligence: an 1H-MRS study of normal human brain. Neuroimage 2005;26:965

44. Filley CM. White matter and behavioral neurology. Ann N Y Acad Sci 2005;1064:162-83

45. Nagy Z, Westerberg $\mathrm{H}$, Klingberg T. Maturation of white matter is associated with the development of cognitive functions during childhood. J Cogn Neurosci $2004 ; 16: 1227-33$

46. Valenzuela MJ, Sachdev PS, Wen W, et al. Dual voxel proton magnetic resonance spectroscopy in the healthy elderly: subcortical-frontal axonal $\mathrm{N}$-acetylaspartate levels are correlated with fluid cognitive abilities independent of structural brain changes. Neuroimage 2000;12:747-56

47. Charlton RA, McIntyre DJ, Howe FA, et al. The relationship between white matter brain metabolites and cognition in normal aging: the GENIE study. Brain Res 2007;1164:108-16. Epub 2007 Jun 21

48. Charlton RA, Barrick TR, McIntyre DJ, et al. White matter damage on diffusion 
tensor imaging correlates with age-related cognitive decline. Neurology 2006;66:217-22

49. Vandermeeren Y, Sebire G, Grandin CB, et al. Functional reorganization of brain in children affected with congenital hemiplegia: fMRI study. Neuroimage 2003;20:289-301

50. Kim SG, Ashe J, Hendrich K, et al. Functional magnetic resonance imaging of motor cortex: hemispheric asymmetry and handedness. Science 1993;261:615-17

51. Chen R, Gerloff C, Hallett M, et al. Involvement of the ipsilateral motor cortex in finger movements of different complexities. Ann Neurol 1997;41:247-54

52. Waber DP, De Moor C, Forbes PW, et al. The NIH MRI study of normal brain development: performance of a population-based sample of healthy children aged 6 to 18 years on a neuropsychological battery. J Int Neuropsychol Soc 2007;13:729-46

53. Ariza M, Junque C, Mataro M, et al. Neuropsychological correlates of basal ganglia and medial temporal lobe NAA/Cho reductions in traumatic brain injury. Arch Neurol 2004;61:541-44

54. Ungerleider LG, Courtney SM, Haxby JV. A neural system for human visual working memory. Proc Natl Acad Sci U S A 1998;95:883-90

55. Smith EE, Jonides JJ, Koeppe RA, et al. Spatial versus object working memory: PET investigations. J Cognit Neurosci 1995;7:337-56

56. Klingberg T. Development of a superior frontal-intraparietal network for visuo-spatial working memory. Neuropsychologia 2006;44:2171-77. Epub 2006 Jan 6

57. Baldo JV, Dronkers NF. The role of inferior parietal and inferior frontal cortex in working memory. Neuropsychology 2006;20:529-38.

58. Hagino H, Suzuki M, Mori K, et al. Proton magnetic resonance spectroscopy of the inferior frontal gyrus and thalamus and its relationship to verbal learning task performance in patients with schizophrenia: a preliminary report. Psychiatry Clin Neurosci 2002;56:499-507

59. Mahone EM, Martin R, Kates WR, et al. Neuroimaging correlates of parent ratings of working memory in typically developing children. J Int Neuropsychol Soc 2009;15:31-41

60. Moore GJ. Proton magnetic resonance spectroscopy in pediatric neuroradiology. Pediatr Radiol 1998;28:805-14

61. Wang Z, Zimmerman RA, Sauter R. Proton MR spectroscopy of the brain: clinically useful information obtained in assessing CNS diseases in children. AJR Am J Roentgenol 1996;167:191-99

62. Saykin AJ, Ahles TA, McDonald BC. Mechanisms of chemotherapy-induced cognitive disorders: neuropsychological, pathophysiological, and neuroimaging perspectives. Semin Clin Neuropsychiatry 2003;8:201-16

63. Castillo M, Kwock L, Courvoisie H, et al. Proton MR spectroscopy in children with bipolar affective disorder: preliminary observations. AJNR Am J Neuroradiol 2000;21:832-38

64. Caetano SC, Fonseca M, Olvera RL, et al. Proton spectroscopy study of the lef dorsolateral prefrontal cortex in pediatric depressed patients. Neurosci Lett 2005;384:321-26

65. Friedman SD, Brooks WM, Jung RE, et al. Proton MR spectroscopic findings correspond to neuropsychological function in traumatic brain injury. AJNR Am J Neuroradiol 1998;19:1879-85

66. Huda A, Guze BH, Thomas A, et al. Clinical correlation of neuropsychological tests with $1 \mathrm{H}$ magnetic resonance spectroscopy in hepatic encephalopathy. Psychosom Med 1998;60:550-56 